J. Clin. Chem. Clin. Biochem.

Vol. 17, 1979, pp. 683-687

\title{
Enzymatische Kreatinin-Bestimmung im Serum: Vergleich mit Jaffé-Methoden
}

Von G. Szász †, U. Börner, E. W. Busch

Institut für Klinische Chemie, Universitätskliniken Giessen

und

W. Bablok

Boehringer Mannheim GmbH, Medizinische Forschung, Mannheim

(Eingegangen am 16. August/8. Oktober 1979)

Zusammenfassung: In 337 Seren wurde die Kreatinin-Konzentration enzymatisch sowie mit 6 verschiedenen Modifikationen der Jaffé-Methode untersucht. Bei zufriedenstellender Präzision des enzymatischen Verfahrens wurden beim Vergleich mit der "Methode nach Adsorption an Fullererde" (Lloyd's Reagenz) übereinstimmende Ergebnisse erhalten. Alle übrigen Pikrat-Methoden gaben zu hohe Werte: Der Median der relativen, prozentualen Differenzen zur enzymatischen Methode lag zwischen $+20 \%$ und $+50 \%$ (Bereich $22-88 \mu$ mol $/ 1$ Kreatinin) bzw. $+10 \%$ und $+30 \%$ (Bereich 89-177 $\mu \mathrm{mol} / 1$ Kreatinin).

Die Ergebnisse bestätigen damit die Unspezifität aller Jaffé-Methoden mit Ausnahme des Verfahrens nach Adsorption an Fullererde.

\section{Enzymatic assay of creatinine in serum: Comparison with Jaffé methods}

Summary: The creatinine concentrations in 337 sera were determined by enzymatic assay and by six different modifications of the Jaffe method. The enzymatic assay showed satisfactory precision and gave the same results as the assay after adsorption on Fuller's earth (Lloyd's reagent). All other methods using picrate gave falsely high values: the relative deviation from the enzymatic method had a median value between $+20 \%$ and $+50 \%$ for creatinine concentrations in the range $22-88 \mu \mathrm{mol} / 1$, and between $+10 \%$ and $30 \%$ for creatinine concentrations in the range $89-177 \mu \mathrm{mol} / 1$.

The study thus confirms the lack of specificity of all Jaffé methods, with the exception of the assay after adsorption on Fuller's earth.

\section{Einftihirung}

Die meisten Methoden zur Bestimmung des Kreatịnins beruhen auf der 1886 beschriebenen Jaffé-Reaktion: Im alkalischen Medium bildet Kreatinin mit Pikrinsäure eine orange L̆ Losung, die photometrisch vermessen werden kann. Diese einfache Methode ist jedoch nicht spezifisch für Kreatinin und deshälb vielfach modifiziert worden: Die kinetische Methode soll weniger störanfällig gegenüber "Jaffé-positiven Substanzen" als die Klassische EndwertMethode sein. Am besten werden Störungen der JafféMethode durch Einsatz von Fullererde (Lloyd's Reagenż) umgangen. Dieses Verfahren gilt als Referenzmethode, ist jedoch aufwendig und wenig praktikabel.
Vor kurzem wurde ein Test eingeführt, der die Bestimmung des Kreatinins auf enzymatischem Wege gestattet. Der Vergleich der verschiedenen Modifikationen der JafféMethode mit der enzymatischen Methode ist Gegenstand dieser Arbeit (1).

\section{Material und Methoden \\ Präzision}

Die Präzision in der Serie und von Tag zu Tag wurde für alle Konzentrationsbereiche und Methoden am selben Untersuchungsmaterial ermittelt. Dazu wurden Fünffach-Analysen je eines Poolserums aus 3 Konzentrationsbereichen an 5 Tagen 
Tab. 1. Methoden, Reagenzien, Geräte und Meßbedingungen.

\begin{tabular}{|c|c|c|}
\hline Methoden und Reagenzien & Geräte & Messung \\
\hline $\begin{array}{l}\text { Enzy matisch nach Wahlefeld et al. (4) } \\
\text { (Test-Combination Creatinin enzymatisch } \\
\text { Boehringer Mannheim GmbH) }\end{array}$ & $\begin{array}{l}\text { Eppendorf-Substratautomat } \\
5032\end{array}$ & 334 nm gegen Probenleerwert (siehe 1.c. (14)) \\
\hline \multicolumn{3}{|l|}{ Modifikationen der Jaffé-Methoden } \\
\hline \multicolumn{3}{|l|}{$\begin{array}{l}\text { Endpunktmethoden } \\
\text { (mit Enteiweißung) }\end{array}$} \\
\hline $\begin{array}{l}\text { Fullererde-Methode nach Knoll \& Stamm (5) } \\
\text { mod. nach Haeckel (pers. Mitt.) }\end{array}$ & Eppendorf $1101 \mathrm{M}$ & 509 nm gegen Reagenzienleerwert \\
\hline $\begin{array}{l}\text { Popper et al. mod. (6) (Test-Combination } \\
\text { Creatinin, Boehringer Mannheim GmbH) }\end{array}$ & Eppendorf 5032 & $\begin{array}{l}509 \text { nm gegen Reagenzienleerwert, nach Vor- } \\
\text { schrift des Herstellers }\end{array}$ \\
\hline Chasson (7), Rapoport (8) & SMA $6 / 60$ & $505 \mathrm{~nm}$, nach Vorschrift des Herstellers \\
\hline Kinetische Methoden & $"$ & \\
\hline Bartels et al. (9) mod. nach Helger et al. (10) & Eppendorf Enzymautomat 5020 & $\begin{array}{l}2 \text { min-Messung bei } 509 \mathrm{~nm} \text { gegen Standard } \\
\text { (siehe l.c. (14)) }\end{array}$ \\
\hline $\begin{array}{l}\text { Adaptation auf IL } 919 \text { Glucose-Harnstoff- } \\
\text { Creatinin-Analyzer }\end{array}$ & $\begin{array}{l}\text { IL } 919 \text { Glucose-Harnstoff- } \\
\text { Creatinin-Analyzer }\end{array}$ & $\begin{array}{l}16 \mathrm{~s} \text {-Messung bei } 525 \mathrm{~nm} \text { nach Vorschrift des } \\
\text { Herstellers; Kalibrierung mit albuminhaltigem } \\
\text { Standard ( } 354 \mu \mathrm{mol} / 1 \text { Kreatinin) }\end{array}$ \\
\hline $\begin{array}{l}\text { Adaptation auf CentrifiChem } 300 \\
\text { (Test-Combination Creatinin, Boehringer } \\
\text { Mannheim GmbH) }\end{array}$ & CentrifiChem 300 & 60 s-Messung nach Vorschrift des Herstellers \\
\hline
\end{tabular}

Tab. 2. Präzisionen der Methoden mit Humanseren in verschiedenen Konzentrationsbereichen $(\mathrm{N}=25)$.

\begin{tabular}{|c|c|c|c|c|c|c|c|c|c|}
\hline \multirow[b]{2}{*}{ Methoden } & \multicolumn{3}{|c|}{$\begin{array}{l}\text { Kreatinin } \\
\text { Niedriger Konzentrationsbereich }\end{array}$} & \multicolumn{3}{|c|}{$\begin{array}{l}\text { Kreatinin } \\
\text { Mittlerer Konzentrationsbereich }\end{array}$} & \multicolumn{3}{|c|}{$\begin{array}{l}\text { Kreatinin } \\
\text { Hoher Konzentrationsbereich }\end{array}$} \\
\hline & $\begin{array}{l}\text { Mittelwert } \\
\text { [ } \mu \mathrm{mol} / \mathrm{l}]\end{array}$ & $\begin{array}{l}\text { VK }[\% \\
\text { Serie }\end{array}$ & v.Tag zu Tag & $\begin{array}{l}\text { Mittelwert } \\
\text { [ } \mu \mathrm{mol} / \mathrm{l}]\end{array}$ & $\begin{array}{l}\text { VK } 1 \% \\
\text { Serie }\end{array}$ & v. Tag zu Tag & $\begin{array}{l}\text { Mittelwert } \\
{[\mu \mathrm{mol} / \mathrm{l}]}\end{array}$ & $\begin{array}{l}\text { VK } 1 \% \\
\text { Serie }\end{array}$ & v.Tag zu Tag \\
\hline Enzymatisch & 71 & 6,0 & 10.7 & 244 & 1,5 & 2,5 & 529 & 1,4 & $.2,4$ \\
\hline $\begin{array}{l}\text { Knoll \& Stamm } \\
\text { Popper et al. mod. } \\
\text { Chasson, Rapoport } \\
\text { (Adaptation auf SMA) }\end{array}$ & $\begin{array}{l}71 \\
75 \\
75\end{array}$ & $\begin{array}{l}2,5 \\
1,2 \\
3,7\end{array}$ & $\begin{array}{l}3,0 \\
2,0 \\
4,6\end{array}$ & $\begin{array}{l}245 \\
246 \\
247\end{array}$ & $\begin{array}{l}1,3 \\
0,4 \\
1,3\end{array}$ & $\begin{array}{l}1,5 \\
1,1 \\
2,0\end{array}$ & $\begin{array}{l}525 \\
523 \\
533\end{array}$ & $\begin{array}{l}1,2 \\
0,4 \\
1,2\end{array}$ & $\begin{array}{l}1,3 \\
1,0 \\
1,7\end{array}$ \\
\hline $\begin{array}{l}\text { Bartels et al. mod. } \\
\text { Adaptation auf IL } 919 \\
\text { Adaptation auf CentrifiChem } \\
300\end{array}$ & $\begin{array}{l}81 \\
90 \\
96\end{array}$ & $\begin{array}{l}5,0 \\
3,4 \\
2,6\end{array}$ & $\begin{array}{l}6.8 \\
5,7 \\
2,8\end{array}$ & $\begin{array}{l}254 \\
265 \\
270\end{array}$ & $\stackrel{1,4}{\overline{0}^{+}}+$ & $\overline{-}_{1,8}^{1,9}+$ & $\begin{array}{l}539 \\
564 \\
552\end{array}$ & $\begin{array}{l}0,8 \\
0,9 \\
2,0\end{array}$ & $\begin{array}{l}1,4 \\
3,2 \\
2,1\end{array}$ \\
\hline
\end{tabular}

+ nicht auswertbar

Tab. 3. Präzisionen der Methoden mit Kontrollseren $(N=25)$.

\begin{tabular}{|c|c|c|c|c|c|c|}
\hline \multirow[b]{2}{*}{ Methoden } & \multicolumn{3}{|c|}{ Kreatinin Precinorm S } & \multicolumn{3}{|c|}{ Kreatinin Monitrol II } \\
\hline & $\begin{array}{l}\text { Mittelwert } \\
{[\mu \mathrm{mol} / \mathrm{l}]}\end{array}$ & $\begin{array}{l}\text { VK }[\%]^{-} \\
\text {Serie }\end{array}$ & $\begin{array}{l}\text { VK [\%] } \\
\text { v.Tag zu Tag }\end{array}$ & $\begin{array}{l}\text { Mittelwert } \\
{[\mu \mathrm{mol} / 1]}\end{array}$ & $\begin{array}{l}\text { VK [\%] } \\
\text { Serie }\end{array}$ & $\begin{array}{l}\text { VK[\%] } \\
\text { v.Tag zu Tag }\end{array}$ \\
\hline Enzymatisch & 130 & 2,9 & 4,8 & 512 & 0,9 & 2,4 \\
\hline $\begin{array}{l}\text { Knoll \& Stamm } \\
\text { Popper et al. mod. } \\
\text { Chasson, Rapoport } \\
\text { (Adaptation auf SMA) }\end{array}$ & $\begin{array}{l}131 \\
130 \\
144\end{array}$ & $\begin{array}{l}1,7 \\
1,0 \\
1,6\end{array}$ & $\begin{array}{r}3,4 \\
1,5 \\
3,9 \\
.\end{array}$ & $\begin{array}{l}505 \\
502 \\
525\end{array}$ & $\begin{array}{l}0,7 \\
0,6 \\
1,5\end{array}$ & $\begin{array}{l}1,1 \\
1,2 \\
2,4\end{array}$ \\
\hline $\begin{array}{l}\text { Bartels et al. mod. } \\
\text { Adaptation auf IL } 919 \\
\text { Adaptation auf CentrifiChem } 300\end{array}$ & $\begin{array}{l}157 \\
156 \\
157\end{array}$ & $\begin{array}{l}3,6 \\
1,8 \\
1,3\end{array}$ & $\begin{array}{l}3,6 \\
4,9 \\
2,6\end{array}$ & $\begin{array}{l}541 \\
538 \\
549\end{array}$ & $\begin{array}{l}0,9 \\
0,8 \\
0,8\end{array}$ & $\begin{array}{l}1,5 \\
3,7 \\
2,4\end{array}$ \\
\hline
\end{tabular}


durchgeführt sowie zwei kommerzielle Kontrollseren eingesetzt. Die Werte wurden auf Normalverteilung geprüft (2) und die Präzisionen mit einer einfachen Varianzanalyse bestimmt (3).

Vergleich suntersuchungen

337 tiefgefrorene Seren des Konzentrationsbereiches von 0-884 $\mu \mathrm{mol} / \mathrm{I}$ Kreatinin wurden portionsweise aufgeteilt. Der Kreatiningehalt wurde mit der enzymatischen Methode sowie 6 Jaffé-

Methoden in Doppelbestimmungen gemessen (Tab. 1). Für den Vergleich wurde der Mittelwert aus beiden Einzelbestimmungen eingesetzt. Die Prüfung auf systematische Unterschiede zwischen enzymatischer Methode und den Vergleichsmethoden erfolgte mit der von Wilcoxon modifizierten Rangvarianzanalyse (Irrtumswahrscheinlichkeit $\alpha=0,05$ ) (11).

\section{Ergebnisse und Diskussion}

Die Tabellen 2 und 3 enthalten die Ergebnisse der Präzisionsuntersuchungen in Human- und Kontrollseren:

Im niedrigen Konzentrationsbereich erreicht die enzymatische Methode nicht die Präzision der Jaffé-Methoden, ist jedoch mit einem Variationskoeffizienten von $6 \%$ in der Serie noch tolerabel. Bei höheren Konzentrationen sind keine großen Unterschiede zwischen den Methoden festzustellen: die Präzisionen in der Serie und von Tag zu Tag liegen für Human- und Kontrollseren meist unter $3 \%$.

\section{Vergleichsuntersuchungen} Vergleichsuntersuchungen der enzymatischen Methode gegenüber den Jaffé-Methoden wiedergegeben und die $90 \%$ Bereiche der prozentualen Differenzen (5. bis 95. Quantil) eingetragen. Zur Ermittlung wurde von nicht klassifizierten Daten ausgegangen (12).

Im niedrigen Konzentrationsbereich (bis $177 \mu \mathrm{mol} / 1$ Kreatinin, Abb. 1) gibt nur die "Jaffé-Methode nach Adsorption an Fullererde" vergleichbare Werte. Es bestehen nur Unterschiede in der Variabilität der beiden Methoden. Der Vergleich mit den übrigen Jaffé-Methoden zeigt, daß wesentlich zu hohe Kreatinin-Konzentrationen vorgetäuscht werden: Der Median der Differenzen zwiVerfahren liegt zwischen etwa $+20 \%$ und $+50 \%$ (Bereich $22-88 \mu \mathrm{mol} / \mathrm{l}$ Kreatinin) bzw. $+10 \%$ und $+30 \%$ (Bereich 89-177 $\mu \mathrm{mol} / 1 \mathrm{Kreatinin}$ ) (Tab. 4).

Die Unterșchiede zu allen Vergleichsmethoden sind die "Fullererde-Methode" ausgenommen - bis zu einer Kreatininkonzentration von $354 \mu \mathrm{mol} / 1$ statistisch signifikant (Tab. 5). Eine Umrechnung der Ergebnisse der Jaffé-Methoden auf die "Fullererde-Methode" oder die enzymatische Methode ist nicht möglich, da die Streuung der Differenzen sehr groß ist: Für den erstgenannten Bemeisten Methoden bei etwa $60 \%$; im Bereich bis $177 \mu \mathrm{mol} / \mathrm{l}$ Ǩreatinin können die Differenzen der JafféMethoden gegenüber der enzymatischen Methode immer noch etwa $+30 \%$ betragen.

\section{Präzision}

In Abbildung 1 und Tabelle 4 sind die Ergebnisse der schen den Vergleichsmethoden und dem enzymatischen reich bis $88 \mu \mathrm{mol} / \mathrm{l}$ Kreatinin liegt das 95 . Quantil für die

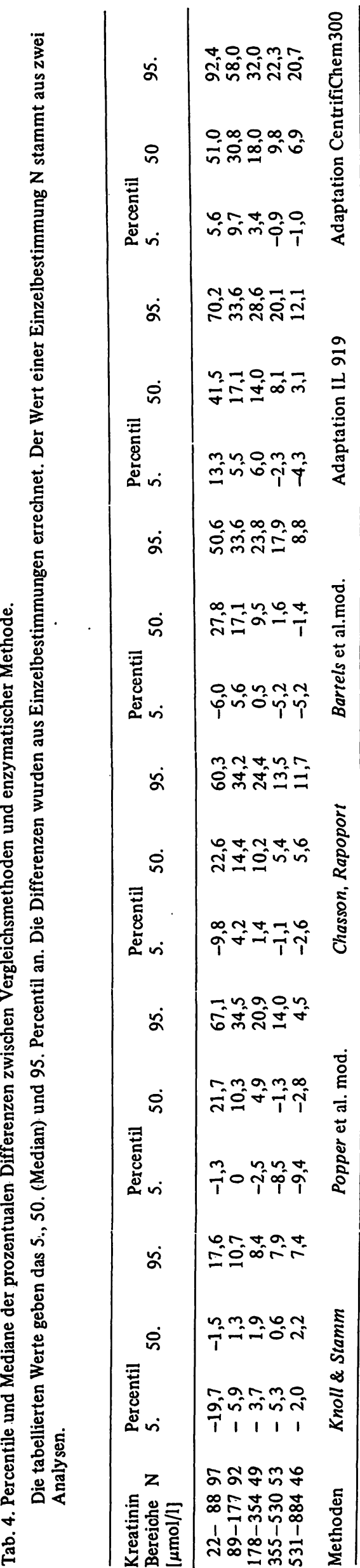

J. Clin. Chem. Clin. Biochem. / Vol. 17, 1979 / No. 11 


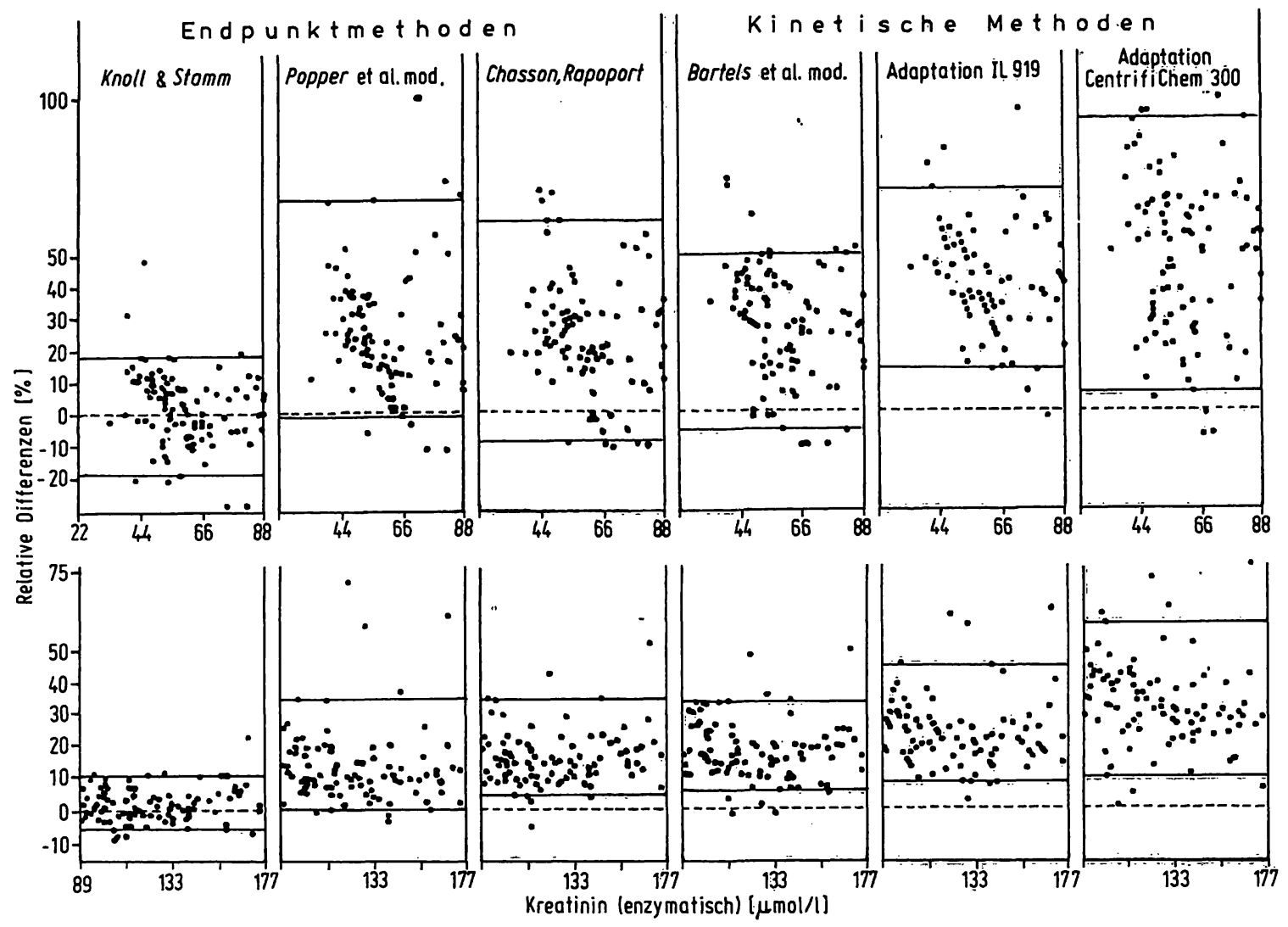

Abb. 1. Differenzen zwischen Vergleichsmethoden und enzymatischer Kreatinin-Bestimmung in \% (Bereich 22-177 $\mu \mathrm{mol} / \mathrm{l}$ ).

Tab. 5. Unterschiede zwischen der enzymatischen Methode und den Vergleichsmethoden $(\mathrm{s}=$ signifikant, $\mathrm{ns}=$ nicht signifikant; Irrtumswahrscheinlichkeit $\alpha=0,05$ ).

Konzentrationsbereiche von Kreatinin [ $\mu \mathrm{mol} / 1]$

\begin{tabular}{llllll} 
Methoden & $22-88$ & $89-177$ & $178-354$ & $355-530$ & $531-884$ \\
\hline Knoll \& Stamm & $\mathrm{ns}$ & $\mathrm{ns}$ & $\mathrm{ns}$ & $\mathrm{ns}$ & $\mathrm{ns}$ \\
Popper et al. mod. & $\mathrm{s}$ & $\mathrm{s}$ & $\mathrm{s}$ & $\mathrm{ns}$ & $\mathrm{ns}$ \\
Chasson, Rapoport (Adaptation auf SMA) & $\mathrm{s}$ & $\mathrm{s}$ & $\mathrm{s}$ & $\mathrm{s}$ & $\mathrm{s}$ \\
Bartels et al. mod. & $\mathrm{s}$ & $\mathrm{s}$ & $\mathrm{s}$ & $\mathrm{s}$ & $\mathrm{ns}$ \\
Adaptation auf IL 919 & $\mathrm{~s}$ & $\mathrm{~s}$ & $\mathrm{~s}$ & $\mathrm{~s}$ & $\mathrm{~ns}$ \\
Adaptation auf CentrifiChem 300 & $\mathrm{~s}$ & $\mathrm{~s}$ & $\mathrm{~s}$ & $\mathrm{~s}$ & $\mathrm{~s}$ \\
\hline
\end{tabular}

Im höheren Konzentrationsbereich werden die Differenzen der Jaffé-Methoden gegenüber der "FullererdeMethode" oder der enzymatischen Methode zunehmend geringer, die relative Streuung der Einzelwerte nimmt mit zunehmender Konzentration ab. Die Unterschiede sind in diesem Bereich jedoch klinisch weniger bedeutungsvoll.

Die Ergebnisse bestätigen die Unspezifität aller PikratMethoden mit Ausnahme des Verfahrens nach Adsorption an Fullererde. Kinetische Methoden oder continuous
flow-Methoden, die mit Dialyse arbeiten, sind ähnlich störanfällig wie Methoden nach Enteiweißung. Die enzymatische und die "Fullererde-Methode" dürften am ehesten die Messung der "wahren" Kreatinin-Konzentration ermöglichen.

Ein wünschenswertër zusätzlicher Vergleich mit einer unabhängigen, spezifischen Mèthode, z.B. Massen-Fragmentographie (13), konnte aus Gründen der Praktikabilität nicht durchgeführt werden. 


\section{Literatur}

1. Szász, G., Börner, U., Bablok, W. \& Busch, E. W. (1978), Clin. Chem. 24, 997 Abstract 047.

2. Conover, W. J. (1971), Practical Nonparametric Statistics, John Wiley \& Sons: New York.

3. Ostle, B. (1966), Statistics in Research, The lowa State University Press, Ames lowa, 2nd edition.

4. Wahlefeld, A. W., Herz, G. \& Bergmeyer, H. U. (1972), Scand. J. Clin. Lab. Inv. 29, Suppl. 126.

5. Knoll, E. \& Stamm, D. (1970), diese Z. 8, 582-587.

6. Popper, H., Mandel, E. \& Mayer, H. (1937), Biochem. Z. 291, 354-367.

7. Chasson, A. L., Grady, H. J. \& Stanley, M. A. (1961), Amer. J. Clin. Pathol. 35, 83-88.
8. Husdan, H. \& Rapoport, A., (1968), Clin. Chem. 14, 222-238. 9. Bartels, H., Böhmer, M. \& Heierli, C. (1972), Clin. Chim. Acta 37, 193-197.

10. Helger, R., Rindfrey, H. \& Hilgenfeld, J. (1974), diese Z. 12, 344-349.

11. Wilcoxon, F. \& Wilcox, R. (1964), Some rapid approximate statistical procedures, Lederle Lab., Pearl River, N.Y., S. 11.

12. Linder, A. \& Berchtold, W. (1979), Elementare statistische Methoden, Birkhäuser Verlag Boston, Stuttgart, S. 21.

13. Björkhem, J., Blomstrand, R. \& Ohman, G. (1977), Clin. Chem. 23, 2114-2121.

14. Börner, U., Szász, G., Bablok, W. \& Busch, E. W. (1979), J. Clin. Chem. Clin. Biochem. 17, 679-682.

Prof. Dr. E. W. Busch Boehringer Mannheim GmbH Diagnostica Forschung Sandhofer Straße 116 D-6800 Mannheim 31 
